\title{
Remarkable recovery in a patient with intradural extramedullary haemangiopericytoma: a case report and literature review
}

\author{
Hongxiang Wang ${ }^{1^{*}}$, Liangzhe Wang ${ }^{2^{*}}$, Yicheng $\mathrm{Lu}^{1}$, Chengyin $\mathrm{Lu}^{1}$, Tao $\mathrm{Xu}^{1}$, Yong Yan ${ }^{1}$, Juxiang Chen ${ }^{1}$ \\ ${ }^{1}$ Department of Neurosurgery, Changzheng Hospital, Second Military Medical University, Shanghai, ${ }^{2}$ Department of Pathology, \\ Changzheng Hospital, Second Military Medical University, Shanghai, China \\ *The authors contributed equally to this work.
}

\begin{abstract}
Haemangiopericytomas (HPCs) in the spinal canal are extremely rare and have only been infrequently reported. Severe and sustained spinal cord compression resulting from these intraspinal tumours may cause potentially irreversible neurological impairment. We described a rare case of primary intradural extramedullary HPC recovered from serious compression of thoracic spinal cord in a 65-year-old man suffered from a rapidly progressive disease with complete paralysis of the lower limbs. Magnetic resonance imaging (MRI) and spinal angiography exhibited an unusual hypervascular mass, which was confirmed as HPC by pathological examination, in the dorsal aspects of $T 7$ to T8 spine. Total surgical resection was performed followed by adjuvant radiation. Moreover, this patient had been undergoing specific training of lower limbs from the onset of paralysis and continuous rehabilitation was conducted after discharge. Increased strength of his lower limb muscles was shown in the period of follow-up. Remarkably, four years after surgery, this patient was able to walk with sticks. No signs of local recurrence or metastasis occurred. Conclusively, surgical removal is still the preferred treatment for HPC, and adjuvant radiotherapy and/or chemotherapy may help control tumour recurrence or metastasis. Additionally, it is possible that rehabilitation training potentially promotes neurological function recovery to some extent.
\end{abstract}

Key words: haemangiopericytoma, spinal cord, paralysis, rehabilitation.

\section{Introduction}

Haemangiopericytoma (HPC) is a malignant neoplasm that originates from Zimmermann pericytes surrounding capillaries and venules [13]. Although found in the central nervous system (CNS), HPC rarely occurs in the spine. Until now, approximately 80 cases of intraspinal HPC have been reported, of which only 10 were located in the intradural extramedullary region $[14,15]$. In terms of clinical presentation and imaging studies, HPC in the CNS resembles meningioma; however, it is characterised by high vascularity and aggressiveness and has a strong tendency to recur locally and metastasise [17]. The 2016 the World Health Organisation (WHO) classified this neoplasm as a solitary fibrous tumour (SFT)/HPC of grade II and III, previously diagnosed as HPC and anaplastic HPC, respectively $[11,16]$. 
Usually tumours including primary tumour and metastatic cancer developing within the spinal canal can result in spinal cord compression, the symptoms of which, involving pain, motor weakness, sensory changes, and sphincter dysfunction, can occur together or present in isolation. In such conditions, neurological compromise may worsen gradually, and delays in diagnosis and treatment may cause potentially irreversible neurological impairment [7].

Herein we report a rare case of intradural extramedullary HPC in the thoracic spine with a very good prognosis after a long course of paraplegia.

\section{Case report}

A 65-year-old man was admitted to our hospital with a three-month history of increasing thoracic back pain after a two-year mild course. He also suffered from progressive weakness of lower limbs and sensory disturbance below his xiphoid for two years. History-taking revealed that his neurological symptoms had proceeded to complete paralysis and loss of sensation in both legs 14 months previously. Eighteen months prior to admission, he had been diagnosed with cervical spinal stenosis and undergone a spinal canal decompressive operation at another hospital. However, all the above symptoms had not been alleviated anymore, and worse, during the subsequent three months, he had developed urinary incontinence and constipation. The rest of the history included heart stent angioplasty three years earlier. Neurological examination revealed bilateral 0/5 strength at hip, knee, and ankle levels. Both the lower limbs showed decreased muscle tone but exaggerated deep tendon reflexes. Lower abdominal and cremasteric reflex was absent. Loss of sensation was examined below T8 level, while the skin region at T7-8 level was more sensitive to pain. Babinski sign was positive bilaterally.

Magnetic resonance imaging of the spine showed the mass as hypointense on T1-weighted and hyper-
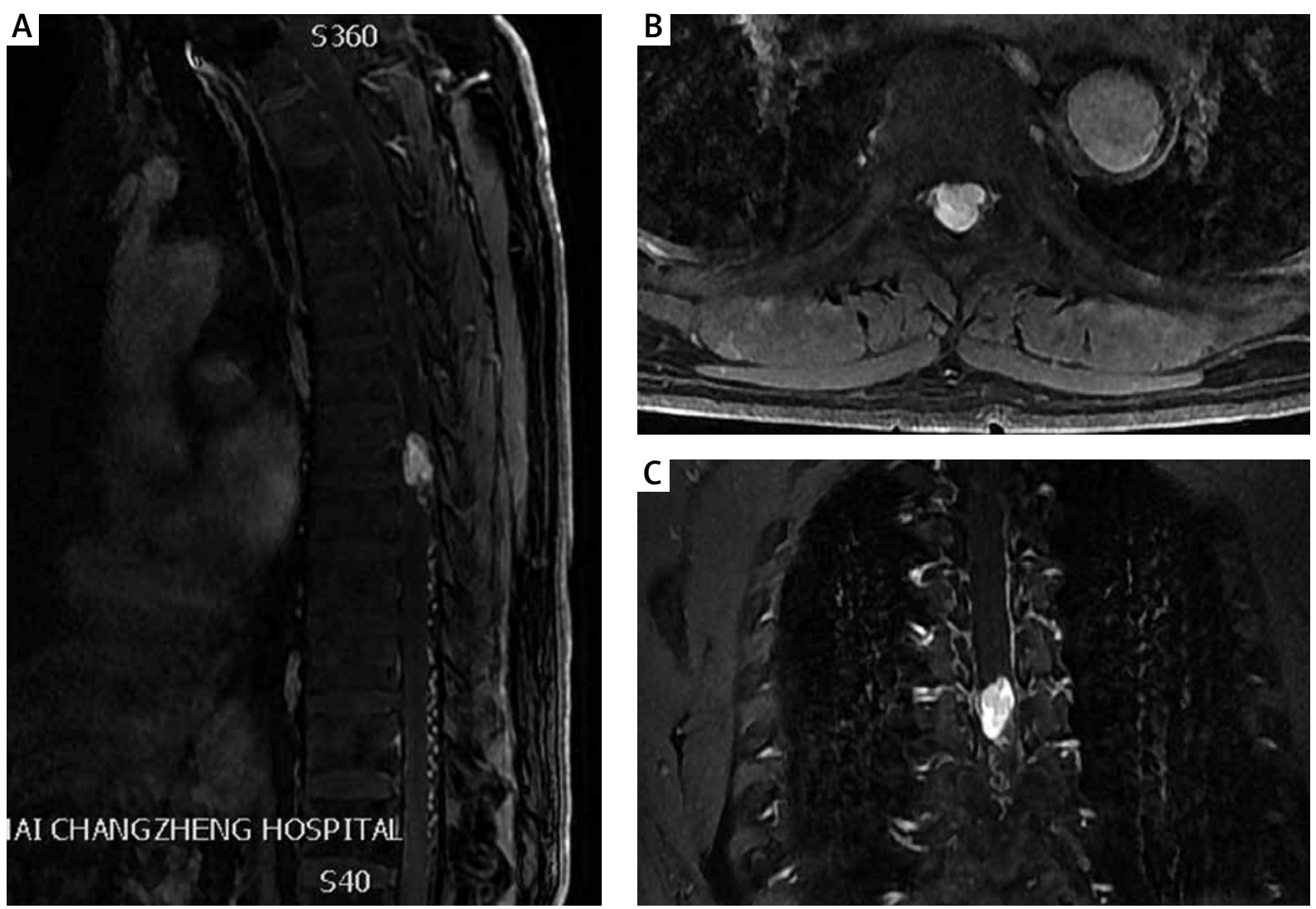

Fig. 1. Contrast-enhanced magnetic resonance imaging scan showing a lesion with intense enhancement in the thoracic spine. The sagittal imaging reveals an intradural mass with radicular venous drainage (A). On the axial and coronal view, this tumour almost occupies the entire space of the spinal canal (B, C). 
intense on T2-weighted images. Contrast-enhanced MRI displayed a well-enhanced intradural extramedullary lesion with severe compression over the cord on the dorsal side at T7-T8 level. Spinal angiography revealed an unusual hypervascular mass with radicular venous drainage and filling of congested intradural veins descending to the level of T12 (Fig. 1).

The patient then underwent T7-T8 laminectomy. Under microscope visualisation, incision of the dura mater revealed an oval, soft, red, and highly vascular tumour with large draining veins beneath it. This mass adhered tightly to the spinal cord, occupied the right dorsolateral gutter and put the cord to the left front. We carefully isolated the tumour border from the cord and performed a complete removal of the intradural extramedullary lesion. A nerve root involved in the tumour was coagulated and severed, as well as the large radicular draining vein identified on the angiogram. The thoracic back pain was significantly alleviated after surgery and the postoperative course progressed without complications.

Microscopically, the intradural extramedullary mass shows abundant blood vessels resulting in
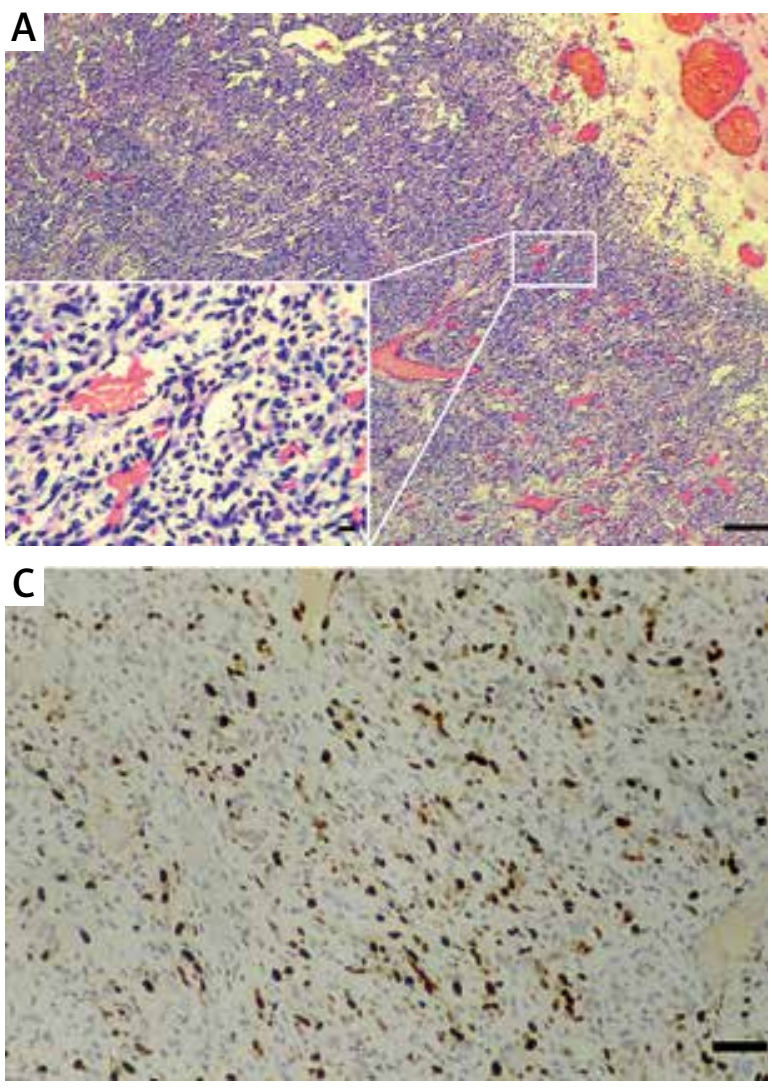

a "staghorn" appearance. Oval to spindled tumour cells with elongated round nuclei and distributed chromatin can be observed as well as occasional mitoses. For immuno-staining, the tumour cells showed positive for CD31, CD34, FVIII, Bcl-2, and Ki67 (25\%), while the negative immune-staining for CD99, smooth muscle actin (SMA), and S-100, which help us rule out the smooth muscle cells, originated or neurogenic tumour (Fig. 2). The tumour cells failed to express STAT- 6 nuclear staining, which is thought to be a specific marker for SFT, but we still made the final diagnosis of HPC/SFT, WHO grade II, after careful observation and consultation by three different experienced pathologists.

With concern about the high risk of recurrence of HPC and the potential benefits of radiotherapy [15], the patient was willing to be suggested for postoperative radiotherapy. The patient then received a onemonth course of the adjuvant therapy with a total dose of $60 \mathrm{~Gy}$ and continued rehabilitation involving skin care, muscle massage, and passive mobility training of lower limbs according to network teaching courses.

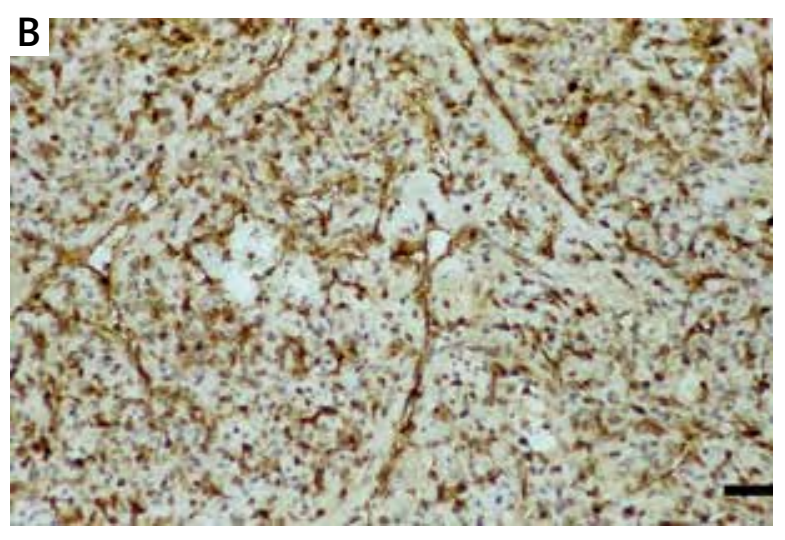

Fig. 2. Haematoxylin and eosin staining (HE, original magnification 40x) shows a highly cellular tumour with typical "staghorn" vascular channels. Photomicrograph with higher magnification (HE, original magnification $200 x$ ) shows proliferation of oval to spindle shaped cells around the vessels and reveals brisk mitotic activity (A). Neoplastic cells are diffusely immunoreactive for CD34 (original magnification 100x) (B). The Ki67 (original magnification 100x) labelling index is about 25\% (C). 

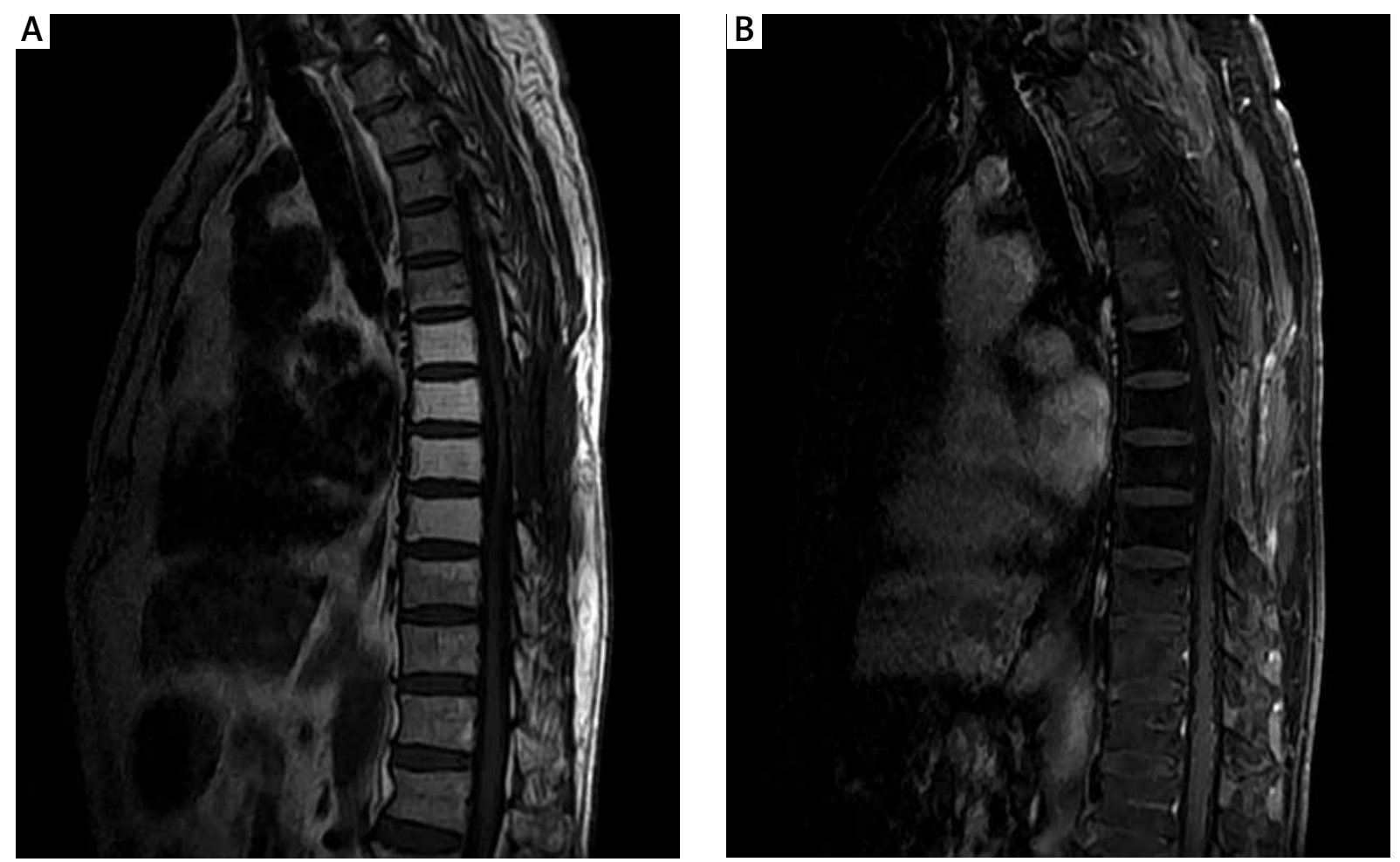

Fig. 3. Sagittal T1-weighted (A) and T1-contrast enhanced (B) images showing no evidence of recurrent disease.

The neurological status of the patient gradually improved. He presented with increased strength of lower limbs, regained pain and warm sense below T8 level, and urinated and defecated normally during a period of 3-5 months. Reflexes had also returned. Four years of follow-up survey indicated that this patient was able to walk with the help of walking sticks and his lower limb muscle strength was up to $4 / 5$. Enhanced MRI examination showed no tumour relapse findings, and no evidence of any symptoms and signs associated with metastatic lesion was found (Fig. 3).

\section{Discussion}

Haemangiopericytoma was first described by Stout and Murray in 1942 [30]. It is a highly vascular mesenchymal neoplasm with rapid growth. The average age of patients at primary diagnosis ranges from 32 to 41 years. This tumour occurs most commonly in the subcutaneous soft tissue and skeletal system, of which only $1 \%$ are found in the CNS $[2,6]$. In the CNS, the tumour is more usually found in the cranial cavity, while spinal involvement is rare. Our search of the literature showed few reported cases including only 10 intradural extramedullary HPCs.
Haemangiopericytoma was once thought to represent an aggressive form of angioblastic meningioma [27], due to the similarities in clinical manifestation and imaging findings. The differential diagnosis of HPC and malignant, invasive meningioma can be very difficult. HPC is usually characterised by non-specificity of radiographic findings, including a dural attachment, dural tail sign, an isodense signal or slightly hyperintense on T1- and T2-weighted imaging, a heterogenous enhancement with contrast, and flow voids $[15,28]$. Thus, a correct diagnosis should be mainly based on careful histopathological evaluation, which is essential for appropriate management. Surgery is the primary treatment of choice for spinal HPC. Previous studies demonstrated that gross total resection, if feasible, improved 5 -year local control and disease-free survival compared with a partial resection in CNS HPCs $[6,26]$. Usually, total resection of intraural HPC is relatively easy because of growth into subdural space and localisation. A few HPCs may follow the cord surface vessels to invade cord tissue, which can increase the operative difficulty [4]. However, some reported results showed that total resection did not represent 
a statistically significant survival or recurrence-free benefit $[4,15]$, which indicated that total resection may not be necessary for intraspinal HPCs with high risk. Given that intradural HPC may be characterised by massive bleeding during surgeries [14], preoperative embolisation can help in bleeding control and better removal; however, if severe compression already exists $[5,17,19]$, embolisation may lead to clinical deterioration, probably caused by tumour swelling [4]. Adjuvant radiation therapy was found to increase local control rates and improve length of survival for CNS HPCs $[9,12,17,26,29]$. However, the results from some other studies showed that patients could not obtain any survival benefits from radiotherapy which also had no likelihood of reduced recurrence rate $[10,15]$. It is difficult to draw conclusions regarding the efficacy of radiotherapy in the treatment of CNS HPCs, and large-scale, prospective studies are needed in the future. More importantly, long-term follow-up and serial imaging is necessary for all patients after surgery regardless of radiation therapy. The role of chemotherapy in the management of HPC is also less clear that previous studies has generally proven this treatment modality ineffective $[2,10]$, while combination therapy with temozolomide and bevacizumab has recently been demonstrated as a generally well-tolerated and clinically beneficial regimen [23]. Additionally, salvage chemotherapy in recurrent HPC seems to be acceptable, with modest efficacy [3]. Currently, survival time and recurrence of patients with intraspinal HPC has been found to be clearly associated with tumour pathological grade, of which high-grade ones had a poor prognosis [15]. P53 expression has also been reported to be an independent protective factor for progression-free survival (PFS), but adjuvant radiation was found to extend PFS only in the p53-negative expression group [31]. The prognostic significance of other molecular biomarkers, such as Ki-67, for those tumours has not been found [31]. Additionally, extradural lesions appeared to relapse earlier than intradural tumours at 2.6 vs. 6 years, respectively [28]. On the whole, recent series reported 5-year survival rates of CNS HPCs ranging from $76 \%$ to $93 \%$, and recurrence could occur many years after initial treatment with a rate of $48 \%$ to $92 \%$ $[15,18,26]$. CNS and/or distant metastasis can occur in $4 \%$ to $50 \%$ of patients in a long time $[18,26]$.

In terms of clinical outcome, patients with HPC have a longer overall survival than other CNS malig- nancies such as glioma [22]. Thus, besides prolonging HPC patients' lifespan, we should also focus on improving the quality of life. Rehabilitation is indicated in all patients with neurological disturbance, and the task is particularly important because patients with spinal tumours, who participate in rehabilitation programs, show general improvement in function, mood, quality of life, and survival, and these benefits can persist until the patient's death $[24,25]$. Furthermore, specialist spinal rehabilitation units are highly recommended to better manage the consequences of spinal cord damage and to achieve the best outcomes [20]. Individual rehabilitation programs should be designed based on the degree of paralysis, the stage of the underlying malignant disease, disturbances in the function of other systems, the patient's age, his/ her expectation, and other factors. For example, paraplegic patients with bowel and bladder incontinence can receive instruction in transfers, incentive spirometry, nutrition, bowel and bladder care, and skin care. Also, ambulatory patients can receive strength and mobility training [1].

In the present case, the diagnosis of spinal HPC was delayed by two years after the onset of symptoms, and this period appeared to be too long and the grade of spinal cord damage seemed too high to gain neurological recovery [21], although total surgical resection of the tumour was performed. To our surprise, he gradually recovered from complete paralysis of lower limbs, and the power of both legs reached grade $4 / 5$ at the levels of hip, knee, and ankle, without any neurological deterioration. When backtracking on information before hospitalisation, we found that this patient had been receiving regular, although non-professional, function exercises with a CPM machine and his caregiver to prevent muscle atrophy because he was unable to move his legs. This reveals that rehabilitation excises play a vital role in the recovery of neurological function when the anatomy of spinal cord nerves is intact, which can be explained, at least partly, by the fact that neuronal centres below the level of the lesion exhibit plasticity, and specific training paradigms will provide input and stimulation to make them recover [8]. In addition, this patient with a history of heart stent angioplasty has been regularly taking aspirin for anticoagulation, which may prevent small blood vessels surrounding the compressed spine cord blockage completely and influence regeneration of neurons to some extent. 
In conclusion, we described an extremely rare case of intradural extramedullary HPC, which caused serious compression of the thoracic spinal cord, and this patient recovered from complete paralysis and loss of feeling in lower limbs after a long course. Surgical removal of the lesion is still the preferred treatment for patients with HPC to relieve symptoms, and adjuvant radiotherapy and/or chemotherapy may help control tumour recurrence or metastasis, which remains inconclusive. Notably, rehabilitation training with enhancement of an appropriate proprioceptive input is an important factor that potentially promotes neurological function recovery.

\section{Acknowledgements}

This work was supported by the National Natural Science Foundation of China (81572501).

\section{Disclosure}

\section{Authors report no conflict of interest.}

\section{References}

1. Abrahm JL, Banffy MB, Harris MB. Spinal cord compression in patients with advanced metastatic cancer: "all I care about is walking and living my life". JAMA 2008; 299: 937-946.

2. Bastin KT, Mehta MP. Meningeal hemangiopericytoma: defining the role for radiation therapy. J Neurooncol 1992; 14: 277-287.

3. Chamberlain MC, Glantz MJ. Sequential salvage chemotherapy for recurrent intracranial hemangiopericytoma. Neurosurgery 2008; 63: 720-726.

4. Chou CW, Hsu SP, Lin SC, Chen MH, Shih YH, Lee LS, Lin CF. Primary intradural hemangiopericytoma with intramedullary invasion. J Chin Med Assoc 2009; 72: 536-541.

5. Ciappetta P, Celli P, Palma L, Mariottini A. Intraspinal hemangiopericytomas. Report of two cases and review of the literature. Spine 1985; 10: 27-31.

6. Combs SE, Thilmann C, Debus J, Schulz-Ertner D. Precision radiotherapy for hemangiopericytomas of the central nervous system. Cancer 2005; 104: 2457-2465.

7. Cowap J, Hardy JR, A'Hern R. Outcome of malignant spinal cord compression at a cancer center: implications for palliative care services. J Pain Symptom Manage 2000; 19: 257-264.

8. Dietz V. Neuronal plasticity after a human spinal cord injury: positive and negative effects. Exp Neurol 2012; 235: 110-115.

9. Dufour H, Metellus P, Fuentes S, Murracciole X, Régis J, FigarellaBranger D, Grisoli F. Meningeal hemangiopericytoma: a retrospective study of 21 patients with special review of postoperative external radiotherapy. Neurosurgery 2001; 48: 756-762.

10. Ecker RD, Marsh WR, Pollock BE, Kurtkaya-Yapicier O, McClelland R, Scheithauer BW, Buckner JC. Hemangiopericytoma in the central nervous system: treatment, pathological features, and long-term follow up in 38 patients. J Neurosurg 2003; 98 1182-1187.
11. Fuller GN, Scheithauer BW. The 2007 Revised World Health Organization (WHO) Classification of Tumours of the Central Nervous System: newly codified entities. Brain Pathol 2007; 17: 304-307.

12. Ghia AJ, Allen PK, Mahajan A, Penas-Prado M, McCutcheon IE, Brown PD. Intracranial hemangiopericytoma and the role of radiation therapy: a population based analysis. Neurosurgery 2013; 72: 203-209.

13. Ghose A, Guha G, Kundu R, Tew J, Chaudhary R. CNS Hemangiopericytoma: A Systematic Review of 523 Patients. Am J Clin Oncol 2017; 40: 223-227.

14. Lee CH, Kim KJ, Jahng TA, Kim HJ. Spinal hemangiopericytoma which needed intraoperative embolization due to unexpected bleeding. J Korean Neurosurg Soc 2013; 54: 253-256.

15. Liu HG, Yang AC, Chen N, Yang J, Qiu XG, Zhang JG. Hemangiopericytomas in the spine: clinical features, classification, treatment, and long-term follow-up in 26 patients. Neurosurgery 2013; 72: 16-24.

16. Louis DN, Perry A, Reifenberger G, von Deimling A, FigarellaBranger D, Cavenee WK, Ohgaki H, Wiestler OD, Kleihues P, Ellison DW. The 2016 World Health Organization Classification of Tumors of the Central Nervous System: a summary. Acta Neuropathol 2016; 131: 803-820.

17. Mena H, Ribas JL, Pezeshkpour GH, Cowan DN, Parisi JE. Hemangiopericytoma of the central nervous system: a review of 94 cases. Hum Pathol 1991; 22: 84-91.

18. Moscovici S, Ramirez-DeNoriega F, Fellig Y, Rosenthal G, Cohen JE, Itshayek E. Intradural extramedullary hemangiopericytoma of the thoracic spine infiltrating a nerve root: a case report and literature review. Spine (Phila Pa 1976) 2011; 36: E1534-1539.

19. Muraszko KM, Antunes JL, Hilal SK, Michelsen WJ. Hemangiopericytomas of the spine. Neurosurgery 1982; 10: 473-479.

20. New PW, Marshall R, Stubblefield MD, Scivoletto G. Rehabilitation of people with spinal cord damage due to tumor: literature review, international survey and practical recommendations for optimizing their rehabilitation. J Spinal Cord Med 2017; 40: 213-221.

21. New PW, Reeves RK, Smith E, Eriks-Hoogland I, Gupta A, Scivoletto G, Townson A, Maurizio B, Post MW. International retrospective comparison of inpatient rehabilitation for patients with spinal cord dysfunction: differences according to etiology. Arch Phys Med Rehabil 2016; 97: 380-385.

22. Omuro A, DeAngelis LM. Glioblastoma and other malignant gliomas: a clinical review. JAMA 2013; 310: 1842-1850.

23. Park MS, Patel SR, Ludwig JA, Trent JC, Conrad CA, Lazar AJ, Wang WL, Boonsirikamchai P, Choi H, Wang X, Benjamin RS, Araujo DM. Activity of temozolomide and bevacizumab in the treatment of locally advanced, recurrent, and metastatic hemangiopericytoma and malignant solitary fibrous tumor. Cancer 2011; 117: 4939-4947.

24. Raj VS, Lofton L. Rehabilitation and treatment of spinal cord tumors. J Spinal Cord Med 2013; 36: 4-11.

25. Ruff RL, Ruff SS, Wang X. Persistent benefits of rehabilitation on pain and life quality for nonambulatory patients with spinal epidural metastasis. J Rehabil Res Dev 2007; 44: 271-278.

26. Schiariti M, Goetz P, El-Maghraby H, Tailor J, Kitchen N. Hemangiopericytoma: long-term outcome revisited. J Neurosurg 2011; 114: 747-755. 
27. Scott M, Kellett G, Peale A. Angioblastic meningioma (hemangiopericytoma) of the cerebellar fossa with metastases to the temporal bone and the lumbar spine. Surg Neurol 1974; 2: 35-38.

28. Shirzadi A, Drazin D, Gates M, Shirzadi N, Bannykh S, Fan X, Hunt L, Baron EM, King WA, Kim TT, Johnson JP. Surgical management of primary spinal hemangiopericytomas: an institutional case series and review of the literature. Eur Spine $J 2013$ 22 Suppl 3: S450-S459.

29. Sonabend AM, Zacharia BE, Goldstein H, Bruce SS, Hershman D, Neugut Al, Bruce JN. The role for adjuvant radiotherapy in the treatment of hemangiopericytoma: a surveillance, epidemiology, and end results analysis. J Neurosurg 2014; 120: 300-308.

30. Stout AP, Murray MR. Hemangiopericytoma: a vascular tumor featuring Zimmermann's pericytes. Ann Surg 1942; 116: 26-33.

31. Zeng LC, Wang Y, Wang Y, Han L, Niu Hongquan, Zhang MX, Ke CS, Chen J, Lei T. Analyses of prognosis-related factors of intracranial solitary fibrous tumors and hemangiopericytomas help understand the relationship between the two sorts of tumors. J Neurooncol 2017; 131: 153-161. 